\title{
Der Einfluss von Schmerzwörtern auf die Schmerzverarbeitung
}

\author{
Maria Richter und Thomas Weiß
}

\author{
Schmerzassoziierte Wörter aktivieren die Schmerzmatrix und triggern das aktuelle \\ Schmerzempfinden. Bei chronischen Schmerzpatienten sind diese Effekte stärker \\ ausgeprägt. Physiotherapeuten sollten also einen „schmerz-armen“ Kommunikationsstil \\ mit ihren Patienten pflegen.
}

\begin{abstract}
Schmerz ist per definitionem eine somatosensorische Wahrnehmung mit negativer Valenz. Dies bedeutet, dass ein Mensch Schmerzen fast ausnahmslos als unangenehm empfindet und er so u. a. lernt, Schmerzen zu vermeiden [9]. Gleichzeitig ist bekannt, dass noxische Stimuli nicht per se als schmerzhaft empfunden werden müssen, sondern dies kontextabhängig ist. So gibt es viele Faktoren, welche die Verarbeitung schmerzhafter Reize im Gehirn beeinflussen wie etwa Emotionen, EmpathieGefühle, Aufmerksamkeit, Lern- und Gedächtnisprozesse sowie Erwartungshaltungen [6, 7, 18-20]. Entsprechend hat wohl jeder erlebt, dass er Schmerzen als weniger stark empfindet oder überhaupt nicht wahrnimmt, wenn er gerade abgelenkt oder seine Stimmung ausgesprochen positiv ist. Umgekehrt verstärken eine hohe Aufmerksamkeitsfokussierung auf das Schmerzereignis oder eine negative Stimmungslage unser Schmerzempfinden.
\end{abstract}

\section{Schmerz und Sprache}

Der Einfluss von Sprache auf die Schmerzwahrnehmung wurde bis dato kaum untersucht [8]. Zwar ist über die Rolle der Sprache im Zusammenhang mit Placebo- oder Nocebo-Effekten einiges bekannt, doch die Frage, ob einzelne Wörter die Schmerzwahrnehmung eines Menschen beeinflussen können, wurde seitens der Wissenschaft eher stiefmütterlich behandelt. Dies überrascht, denn wenn Schmerzimpulse nachgewiesenermaßen die Aufmerksamkeit eines Menschen erregen, dessen Gefühlslage beeinflussen und dies Gedächtnisspuren hinterlassen kann, so liegt doch die Vermutung nahe, dass Schmerz unweigerlich und wohl unwillkürlich im Gehirn auch eine semantische Repräsentation, die der Beschreibung des Schmerzes entspricht, also etwa "stechend“ oder „brennend“, ansteuert.

Assoziationen In der Psychologie bedeutet der Begriff Assoziation, dass einfache kognitive Elemente, Emotionen oder Sinneseindrücke unter bestimmten Bedingungen miteinander verknüpft werden können. Dies erklärt das Phänomen, dass zwei oder mehr ursprünglich isolierte psychische Inhalte eine so enge Verbindung miteinander eingehen, dass das Aufrufen eines Assoziationsgliedes das Auftreten eines oder mehrerer weiterer Assoziationsglieder nach sich zieht oder zumindest begünstigt.

Lernprozesse Nach der Geburt besitzt ein Mensch keinerlei Assoziationen zwischen irgendeinem Wort, einem Gefühl oder einem Gegenstand. Erst mit der Zeit lernt er durch seine individuelle Lebenssituation und -erfahrung, Wörter mit Dingen oder Gefühlen zu verknüpfen et vice versa. Im Laufe eines Lebens entstehen so unzählige Gedankenverknüpfungen, die von unterschiedlichen Faktoren beeinflusst werden.

Ein alltägliches Beispiel soll helfen, diese kortikale Kopplung deutlich zu machen.

\section{FALLBEISPIEL}

\section{Kontextabhängige Assoziationen}

Ein Rettungssanitäter oder Notarzt wird beim Hören des Begriffs „Trage“ wahrscheinlich die Trage in einem Rettungsfahrzeug assoziieren, ein Bestatter womöglich an eine „Trag-Bahre“ denken. Je nach Kontext sind auch Assoziationen des Begriffs mit einem „TragGestell“ oder einem „Korb“ möglich. Wiederum anders mögen die gedanklichen Verknüpfungen bei jungen Eltern sein, die mit dem Begriff „Trage“ voraussichtlich an eine „Baby-Trage“ denken - in Abhängigkeit wiederum davon, wie häufig sie eine solche Trage benutzt haben und wie lang diese Erfahrung zurückliegt.

Das Wort „Trage“ ist also assoziativ in unserem Gehirn eingebunden, und die Assoziationsstärke variiert aufgrund der persönlichen Erfahrungen und Lebensumstände. 


\section{Netzwerktheorie}

Gemäß der Netzwerktheorie von Donald Hebb steigert sich die Assoziationsstärke jeglicher Stimuli mit der wiederholten gemeinsamen Erregung der Kontakte zwischen den beteiligten Nervenzellen - dies u.a. durch den Prozess der Langzeitpotenzierung [4].

Assoziative Kopplung Derartige Kopplungen treten auch hinsichtlich der menschlichen Sprachwahrnehmung auf. So aktivieren Verben wie „Springen“ oder „Laufen“ nicht nur jene Neuronen in Hirnbereichen, welche die Sprache verarbeiten, sondern automatisch auch sensomotorische und motorische Areale, in denen die homunkulären Strukturen für das Bein lokalisiert sind [12]. Analog aktivieren Begriffe wie „Küssen“ und „Schmecken“ die entsprechenden Gesichtsregionen des Homunkulus; "Schreiben“ und „Greifen“ die Handareale.

\section{Schmerzmatrix und Sprachareale}

Da jeder Mensch im Laufe seines Lebens wiederholt Erfahrungen mit Schmerz sammelt, sollte es in Analogie zu Hebbs Netzwerkvorstellungen sodann zur engen Kopplung zwischen den neuronalen Strukturen der Schmerzmatrix und ihrer semantischen Repräsentation in den entsprechenden Spracharealen kommen. Dies bedeutet, dass die Erfahrung von „brennendem“ Schmerz unweigerlich die sprachliche Repräsentation von „brennendem Schmerz" anregt.

Gretchen-Frage Spannenderweise könnte im Umkehrschluss davon ausgegangen werden, dass die sprachliche Verarbeitung schmerzassoziierter Wörter nicht nur die Sprachareale des Gehirns sondern darüber hinaus auch Teile der Schmerzmatrix aktiviert [5,21]. Können also verbale Reize die Neuromatrix des Schmerzes aktivieren? Beeinflussen Worte wie „kneifend“, „quetschend“ oder „krampfartig“ unsere Schmerzwahrnehmung und triggern diese?

\section{Neuroanatomische Grundlagen}

Um zu klären, ob „Schmerzwörter“ tatsächlich Schmerzen beeinflussen können und welche Folgen dies hat, ist es wichtig, den Aufbau der Neuromatrix des Schmerzes zu kennen.

Kernstrukturen Die Kernstrukturen der Schmerzmatrix werden durch alle Arten noxischer Reize aktiviert.

Zu ihnen gehören ( $\triangleright$ Abb. 1):

- Rückenmark

- Thalamus

- primäre und sekundäre somatosensorische Kortexareale (S1 und S2)

- posteriore und anteriore Insula

- dorsaler Anteil des anterioren zingulären Kortex (dACC; auch anteriorer midcingulärer Kortex bzw. aMCC [20])

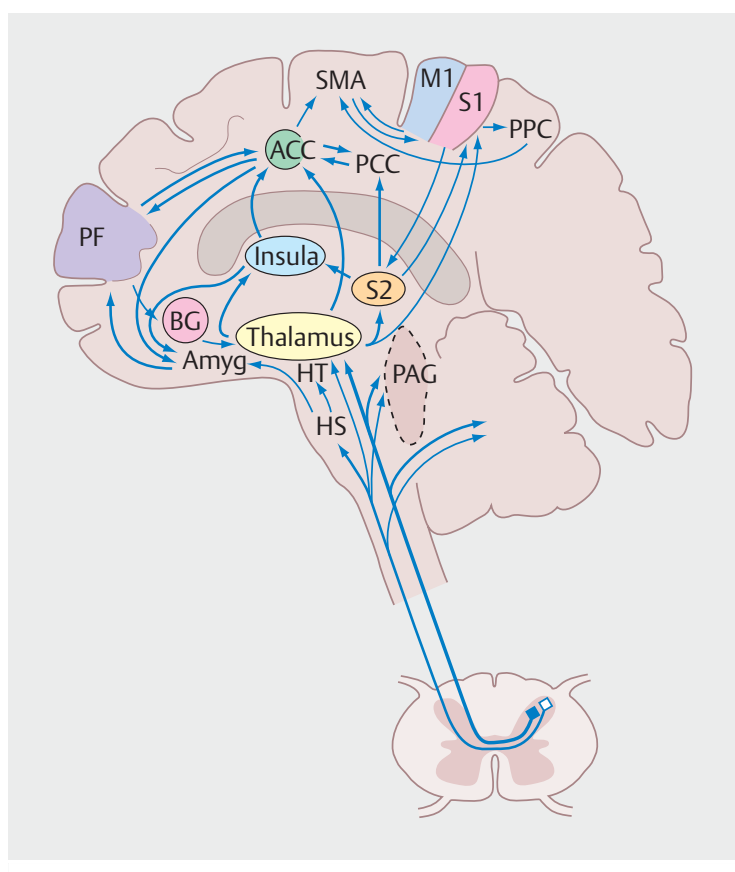

- Abb. 1 Schema zur Verarbeitung noxischer Reize in der Neuromatrix des Schmerzes. Gezeigt sind auch funktionelle Zusammenhänge, die allerdings oft noch nicht eindeutig belegt sind (modifiziert nach [1]): ACC: anteriorer zingulärer Kortex, Amg: Amygdala (Mandelkern), HS: Hirnstamm, HT: Hypothalamus, M1: primärer motorischer Kortex, PAG: periaquäduktales Grau, PCC: posteriorer zingulärer Kortex, PF: präfrontaler Kortex, PPC: posteriorer parietaler Kortex, S1: primärer somatosensorischer Kortex, S2: sekundärer somatosensorischer Kortex, SMA: supplementärmotorisches Feld. Quelle: van den Berg F. Angewandte Physiologie, Band 4, Schmerzen verstehen und beeinflussen. 2. Aufl. Stuttgart: Thieme; 2008.

Erweiterte Schmerzmatrix Die Aktivität der erweiterten Schmerzmatrix ist dagegen situations- und kontextabhängig; sie wird bei noxischen Stimuli also nicht immer aktiviert $[1,11]$. Zu den entsprechenden Strukturen gehören u. a. der präfrontale Kortex (PFC), der posterior parietale Kortex (PPC) oder der Nucleus caudatus.

\section{„Schmerzwörter"}

Um zu belegen, dass die sprachliche Verarbeitung schmerzassoziierter Wörter nicht nur die kortikalen Sprachareale sondern auch Teile der Schmerzmatrix aktiviert, wurde zunächst umfassendes Wortmaterial zusammengetragen. Für die Fahndung nach adäquaten "Schmerzwörtern“ wurde das Corpus Search, Management and Analysis System (COSMAS 3.6.1.) des Instituts für Deutsche Sprache (IDS) in Mannheim genutzt.

Adjektive Ausgewählt wurden Adjektive, die entsprechend ihrer semantischen Bedeutung kategorisiert wurden in „positiv“, „neutral“, „negativ, aber nicht schmerz- 
> Tab. 1 Wortliste mit Adjektiven für die Kategorien „schmerzbezogen“, „negativ“, „,neutral“ sowie „positiv“, die hinsichtlich Wortlänge, Silbenzahl und Häufigkeit im schriftlichen deutschen Sprachgebrauch parallelisiert sind.

\begin{tabular}{|l|l|l|l|}
\hline $\begin{array}{l}\text { schmerzrelevanter } \\
\text { Inhalt }\end{array}$ & $\begin{array}{l}\text { negativer aber nicht- } \\
\text { schmerzrelevanter Inhalt }\end{array}$ & neutraler Inhalt & positiver Inhalt \\
\hline quälend & eklig & gehend & streichelnd \\
\hline lähmend & feindlich & eckig & wärmend \\
\hline zermürbend & intrigant & kurzhaarig & beschwingend \\
\hline peinigend & widerlich & eiförmig & himmlisch \\
\hline plagend & warzig & gewölbt & flirtend \\
\hline kneifend & schimmlig & aschblond & kuschelnd \\
\hline quetschend & stinkend & klappbar & küssend \\
\hline bohrend & verdreckt & kubisch & traubenförmig \\
\hline kolikartig & angsteinflößend & auditiv & becherotisch \\
\hline krampfartig & hasserfüllt & & \\
\hline
\end{tabular}

relevant“ sowie „schmerzrelevant“. Sämtliche Wortkategorien unterschieden sich nicht hinsichtlich Wortlänge, Silbenanzahl und Häufigkeit ihres Vorkommens in der alltäglichen deutschen Sprache.

Anschließend wurden 40 Versuchspersonen gebeten, aus dieser Auswahl von Adjektiven deren Valenz bzw. positive oder negative emotionale Wirkung, das Arousal bzw. die wenig oder stark aktivierende/erregende Wirkung sowie ihre Relation zur Beschreibung von Schmerz zu bewerten. Schließlich wurden dann jene Adjektive extrahiert, die als besonders schmerzbeschreibend eingestuft wurden, ohne dass eine Überschneidung zu den anderen Kategorien entstand.

Valenz Beim Vergleich von schmerzbeschreibenden Adjektiven und negativen Adjektiven mit nicht-schmerzrelevantem Inhalt wurde darauf geachtet, dass sich die Valenz zwischen beiden Gruppen nicht unterschied. Dadurch wurde ausgeschlossen, dass die positive oder negative emotionale Wirkung der Wörter nicht für mögliche Unterschiede in der zentralen Verarbeitung verantwortlich gemacht werden kann. Folglich bekamen die Studienteilnehmer neben Schmerzadjektiven wie „lähmend“, „zermürbend“ und „peinigend“ auch andere negativ besetzte Worte - etwa „eklig“, „schimmlig“ oder „stinkend“ - zu hören.

Die Valenz der Wörter mit positivem Inhalt war vom Vorzeichen umgekehrt, aber vom Betrag nicht unterschiedlich zu schmerzbeschreibenden und negativen Wörtern mit nicht-schmerzrelevantem Inhalt. Schließlich wurde kontrolliert, dass positive, negative und schmerzbeschreibende Adjektive das gleiche mittlere Ausmaß an Aktivierung erzielten.
Stimulationsmaterial Die über dieses komplexe Procedere gefundenen Wörter dienten in den folgenden Untersuchungen als Stimulationsmaterial. Ausgewählt wurden 40 Adjektive, die in die vier Wortkategorien unterteilt wurden ( $\triangleright$ Tab. 1$)$.

\section{Aktivieren schmerzassoziierte Wörter die Schmerzmatrix?}

Wie eingangs dargestellt sollte die während des Lebens stets wiederkehrende Kopplung zwischen dem Erleben von Schmerz und seiner Beschreibung zur assoziativen Verbindung zwischen Wort und Schmerzmatrix führen ähnlich wie bei jenen Verben, die automatisch sensomotorische und motorische Areale aktivieren. Hypothetisch sollte also bereits das alleinige Lesen von schmerzbeschreibenden Adjektiven verschiedene Strukturen der Schmerzmatrix aktivieren.

Versuchsaufbau Zur Verifizierung dieser Hypothese wurden die ausgewählten Adjektive gesunden Versuchspersonen in einem Scanner präsentiert und deren Hirnaktivierung mittels funktioneller Kernspintomografie (fMRT) registriert.

Aufgabenstellung Die Probanden sollten sich Situationen vorstellen, die mit den gezeigten Adjektiven beschreibbar wären. Bei der zweiten Aufgabe sollten sie simultan die Vokale der Wörter zählen ( $\mathbf{A b b}$. 2).

Ergebnisse Für beide Konditionen wurde untersucht, ob es zu einer Aktivierung der Schmerzmatrix kam. Hierbei konnte nachgewiesen werden, dass schmerzassoziierte Adjektive verschiedene Regionen der Schmerzmatrix aktivieren, wenn sich die Testpersonen Situationen vor Augen führen, die durch die Adjektive beschreibbar wären $[15,16]$. 


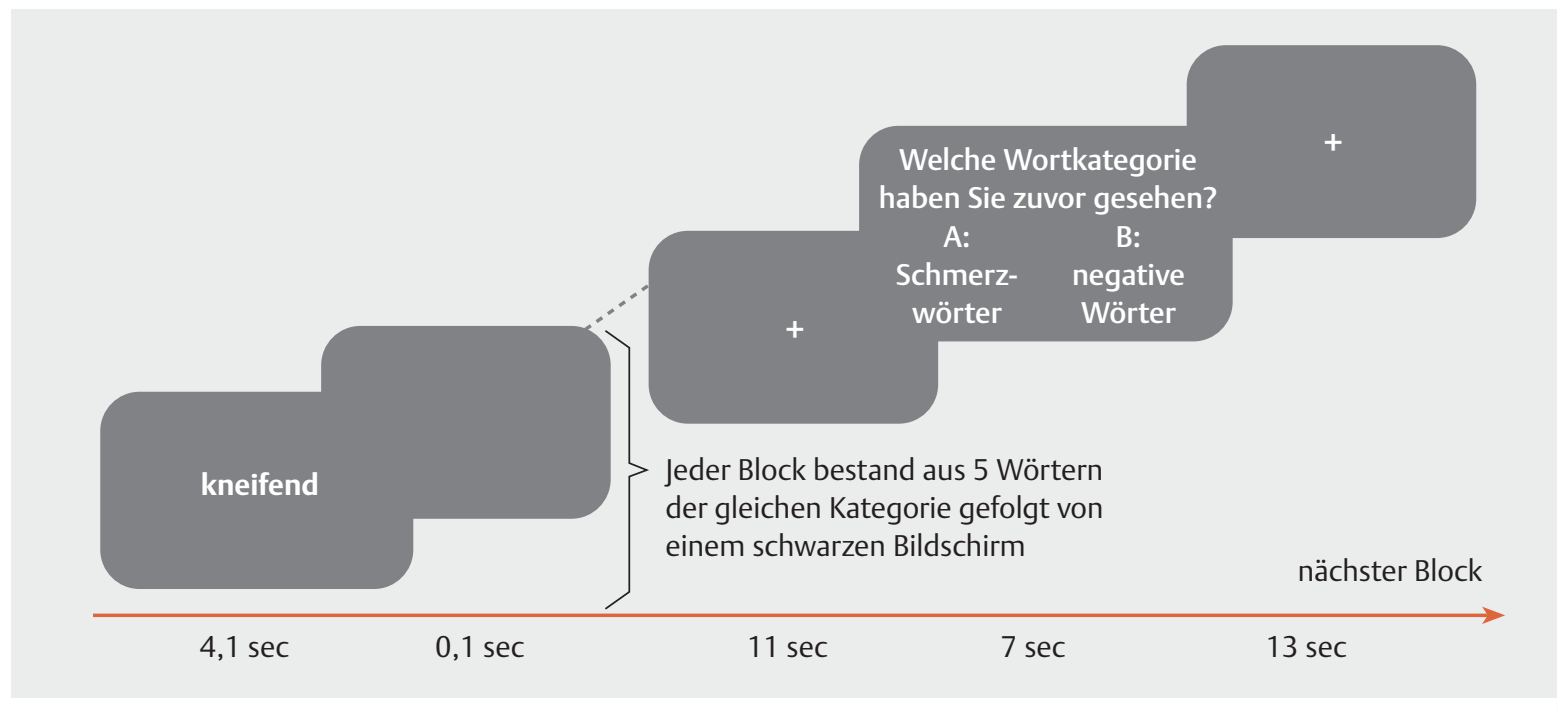

- Abb. 2 Ablauf der Untersuchung im fMRT-Scanner. Quelle: Maria Richter und Thomas Weiß.

So wurden mittels der schmerzbeschreibenden Adjektive sämtliche Kernstrukturen der Schmerzmatrix sowie überdies die erweiterte Schmerzmatrix in den präfrontalen und posterior parietalen Kortizes (PFC bzw. PPC) aktiviert, wenn die Versuchspersonen auf schmerzassoziierte Wörter achteten ( $\vee$ Abb. 1).

Ablenkungsmanöver Selbst für den Fall, dass die Probanden die Vokale der Wörter zählten, damit quasi von deren Inhalt abgelenkt waren, imponierten einige der schmerzspezifischen Aktivierungen in der Neuromatrix.

Ausschlussverfahren Um auszuschließen, dass die Schmerzmatrix allein durch die negative Valenz eines Wortes aktiviert wird, wurde die Verarbeitung negativer Adjektive mit nicht-schmerzrelevantem Inhalt mit jener von schmerzassoziierten Adjektiven verglichen. Als Ergebnis zeigten sich spezifisch für schmerzassoziierte Adjektive höhere Aktivierungen in einigen Teilen der Schmerzmatrix, u.a. in Teilen des präfrontalen Kortex (PFC) sowie des anterioren zingulären Kortex (ACC).

\footnotetext{
Merke

Schmerzwörter aktivieren die Schmerzmatrix Wörter, die mit Schmerz assoziiert werden, aktivieren die Neuromatrix des Schmerzes.

Ein Teil dieser Aktivierung ist nicht ausschließlich auf die negative Valenz dieser Wörter sondern auf ihren schmerzspezifischen Inhalt zurückzuführen.
}

\section{Wirken schmerzassoziierte Wörter stärker bei chronischen Schmerz- patienten?}

Wenn nun die Assoziationsstärke zwischen Schmerzwort und Schmerzmatrix von der Häufigkeit der Assoziationen abhängt, sollte bei chronischen Schmerzpatienten die Neuromatrix des Schmerzes durch schmerzbeschreibende Adjektive intensiver aktiviert werden als bei Gesunden gleichen Alters. Grundlage für diese Annahme ist die Tatsache, dass Patienten mit chronischem Schmerz weitaus häufiger Schmerzerfahrungen machen.

Migränepatienten Um diese Hypothese zu bestätigen, wurden mittels des bereits vorgestellten Versuchsparadigmas chronische Migränepatienten sowie alters- und geschlechts-parallelisierte gesunde Kontrollpersonen untersucht. Die Migräniker waren zum Zeitpunkt der Untersuchung schmerzfrei. Die Schmerzmatrix-Aktivierung bei der kortikalen Verarbeitung schmerzassoziierter Adjektive wurde hierzu erneut mit der Baseline, d. h. ohne jegliche Stimulation, sowie spezifisch auch im Vergleich zur kortikalen Verarbeitung negativer Adjektive ohne schmerzrelevanten Inhalt untersucht [3].

Erhöhte Aktivität Die Vergleiche zeigten für alle Personen wiederholt erhöhte Aktivitäten in Teilen der Schmerzmatrix durch die schmerzbeschreibenden Adjektive, etwa in der posterioren und anterioren Insula oder im sekundären somatosensorischen Kortex (S2) ( $\triangleright$ Abb. 1). Gleichzeitig wurden einige Teile der Neuromatrix des Schmerzes bei den Migränepatienten im Vergleich zu gesunden Kontrollpersonen stärker aktiviert. 
Merke

Schmerzwörter wirken stärker bei chronischen Schmerzpatienten

Bei chronischen Schmerzpatienten wird die Neuromatrix des Schmerzes durch schmerzbeschreibende Adjektive intensiver aktiviert als bei Gesunden gleichen Alters.

\section{Verstärken schmerzassoziierte Wörter die aktuelle Schmerzwahrnehmung?}

In einer Folgeuntersuchung sollte dann geklärt werden, ob ggf. auch die aktuelle Schmerzwahrnehmung bei chronischen Schmerzpatienten durch die Schmerzadjektive beeinflusst wird. Hierfür wurden chronische Schmerzpatienten und parallelisierte Kontrollpersonen dem gleichen Paradigma unterworfen [17].

Erneut zeigte sich, dass bei chronischen Schmerzpatienten mit aktuellen Schmerzen die Schmerzmatrix stärker durch Schmerzwörter aktiviert wird als bei Gesunden. Dieses Ergebnis repliziert én passant die Resultate, die bereits bei den Migränepatienten gefunden wurden und bestätigt erneut, dass schmerzbeschreibende Adjektive bei chronischen Schmerzpatienten die Neuromatrix des Schmerzes stärker aktivieren als bei gesunden Gleichaltrigen.

Darüber hinaus wurde deutlich, dass die Aktivierung in einigen Arealen der Schmerzmatrix wie etwa in der Insula von der aktuellen Schmerzstärke während der Untersuchung abhängig war.

\footnotetext{
Merke

Schmerzwörter verstärken momentane Schmerzen Je stärker ein Mensch unter Schmerzen leidet, desto stärker reagiert seine Schmerzmatrix in diesem Moment auf zusätzliche schmerzassoziierte semantische Reize.
}

\section{Führen schmerzassoziierte Wörter zur Voraktivierung der Schmerzmatrix?}

Wie gezeigt wurde, aktivieren schmerzassoziierte Adjektive Teile der Schmerzmatrix. Wenn einem Probanden nun vor dem Applizieren eines noxischen Stimulus ein Schmerzadjektiv präsentiert wird, könnte dieser physikalische Reiz eine größere Aktivierung in der Schmerzmatrix und damit einen höheren Schmerz verursachen, als wenn vorher ein neutrales Adjektiv gezeigt wird.

Erinnerungstest Um diese Vermutung zu verifizieren, wurde die kortikale Verarbeitung von Adjektiven mit einer tatsächlichen Schmerzstimulation gekoppelt. Dazu wurden den Versuchspersonen auf einem Bildschirm die
Adjektive der vier unterschiedlichen Kategorien angeboten, die sie sich für einen späteren Erinnerungstest merken sollten ( $\triangleright$ Tab. 1). Die Motivation der Probanden zur Wortverarbeitung wurde dadurch erhöht, dass für jedes richtig erinnerte Wort ein finanzieller Bonus in Aussicht gestellt wurde.

Hitze-Stimulation Simultan zur Wortpräsentation erfolgte eine schmerzhafte Hitze-Stimulation mittels eines Laserstimulators. Die applizierten Reize blieben unabhängig von der präsentierten Wortkategorie in ihrer physikalischen Intensität gleich.

NRS und LEP Die subjektive Schmerzhaftigkeit der Laserreize wurde nach jedem Durchgang mittels Numerischer Ratingskala (NRS von $0=$ kein Schmerz bis $100=$ maximal vorstellbarer Schmerz) erfragt. Zusätzlich wurde die evozierte Hirnaktivität auf die Laserstimulation (Laserevozierte Potentiale, LEP), deren Amplituden als objektives Maß der Schmerzempfindung gelten, abgeleitet [10].

Semantisches Priming Im Ergebnis variierte die subjektive Schmerzeinschätzung der Probanden in Abhängigkeit von der Wortkategorie [2]. So traten bei der Verarbeitung schmerzbeschreibender Adjektive im Sinne des semantischen Primings trotz stets identischer HitzeLaserreize signifikant höhere NRS-Werte als auf als bei der Präsentation neutraler Adjektive.

Gleichzeitig waren die Laser-evozierten Potentiale bei Vorreizung durch schmerzbeschreibende Wörter signifikant höher; die Probanden hatten also tatsächlich ein gesteigertes Schmerzempfinden. Die höheren NRS-Werte resultieren also nicht oder nicht ausschließlich daraus, dass die Versuchspersonen möglicherweise den Sinn und Zweck der Studie durchschaut hatten.

\section{Stärkere Effekte bei Chronizität}

Um die stärkeren Effekte bei chronischen Schmerzpatienten nachzuweisen, wurden chronische Migränepatienten mit demselben Untersuchungsdesign untersucht und deren Ergebnisse anschließend mit einer gesunden Kontrollgruppe verglichen [23].

Affektive Verarbeitung Auch hier imponierten bei der kortikalen Verarbeitung schmerzbeschreibender Adjektive höhere NRS-Schmerzratings sowie höhere LEPs auf die gleichstarken Hitzereize. Bei den chronischen Migränikern waren zudem die Schmerzratings und die LEPs tendenziell höher als bei gesunden Kontrollpersonen. Diese Schmerzverstärkung war besonders dann ausgeprägt, wenn die Wörter eher affektiv schmerzbeschreibend waren (z. B. „peinigend“ im Vgl. zu „stechend“). Das Untersuchungsergebnis unterstreicht somit die große Bedeutung der affektiven Verarbeitung von Schmerzen bei chronischen Schmerzpatienten ( $\bullet$ Abb. $\mathbf{3}$ ). 


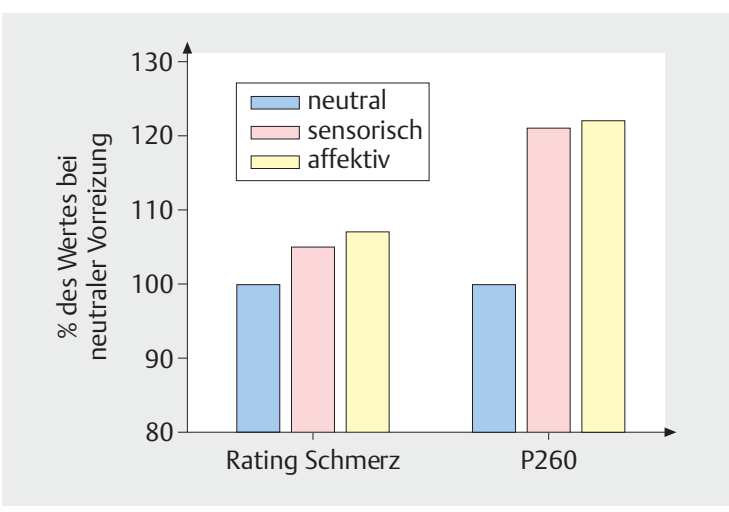

- Abb. 3 Priming von Schmerzwahrnehmung und evozierten Hirnantworten. Im Vergleich zu einem neutralen Vorreiz führt die Vorreizung mit einem schmerzassoziierten Adjektiv trotz gleicher physikalischer Reizintensität zur Zunahme von Schmerzwahrnehmung und evozierter Hirnantwort (gemessen als Amplitude der Komponente P260). Quelle: van den Berg F. Angewandte Physiologie. Band 4, Schmerzen verstehen und beeinflussen. 2. Aufl. Stuttgart: Thieme; 2008.

\section{Merke}

Schmerzwörter triggern die Schmerzwahrnehmung Das Lesen von schmerzbeschreibenden Adjektiven führt zur Voraktivierung von Strukturen der Neuromatrix des Schmerzes, was bei tatsächlichen Schmerzen zu einer stärkeren subjektiven Schmerzempfindung (NRS) und einer messbar erhöhten kortikalen Reaktion (LEP) führt.

Diese Voraktivierung ist bei chronischen Schmerzpatienten stärker ausgeprägt.

\section{Ausblick}

Angesichts der dargestellten Ergebnisse lässt sich eindeutig festhalten, dass die Verarbeitung von schmerzassoziierten Adjektiven nicht nur die entsprechenden Sprachareale sondern auch Regionen der Schmerzmatrix aktiviert. Dies steht in Einklang mit der eingangs erwähnten Theorie neuronaler Zellverbände von Hebb [4].

Teufelskreis Im klinischen Alltag ist die Tatsache, dass schmerzassoziierte Wörter bei chronischen Schmerzpatienten stärker wirken und dass hier die Voraktivierung der Schmerzmatrix durch Schmerzwörter stärker ausgeprägt ist, von großer Tragweite. Tatsächlich deutet sich hier ein perfider Teufelskreis an ( $\boldsymbol{A} \mathbf{A b b} . \mathbf{4})$.

Kontextfaktoren Zweifelsohne sind chronische Schmerzpatienten häufiger Situationen ausgesetzt, in denen sie schmerzassoziierte Kontextfaktoren wie z.B. schmerzbezogene Wörter verarbeiten. Dies geschieht etwa im Rahmen der Visite beim Arzt, Physiotherapeuten oder Heilpraktiker sowie beim Besuch einer Apotheke. Oftmals reicht bei chronischen Schmerzpatienten schon

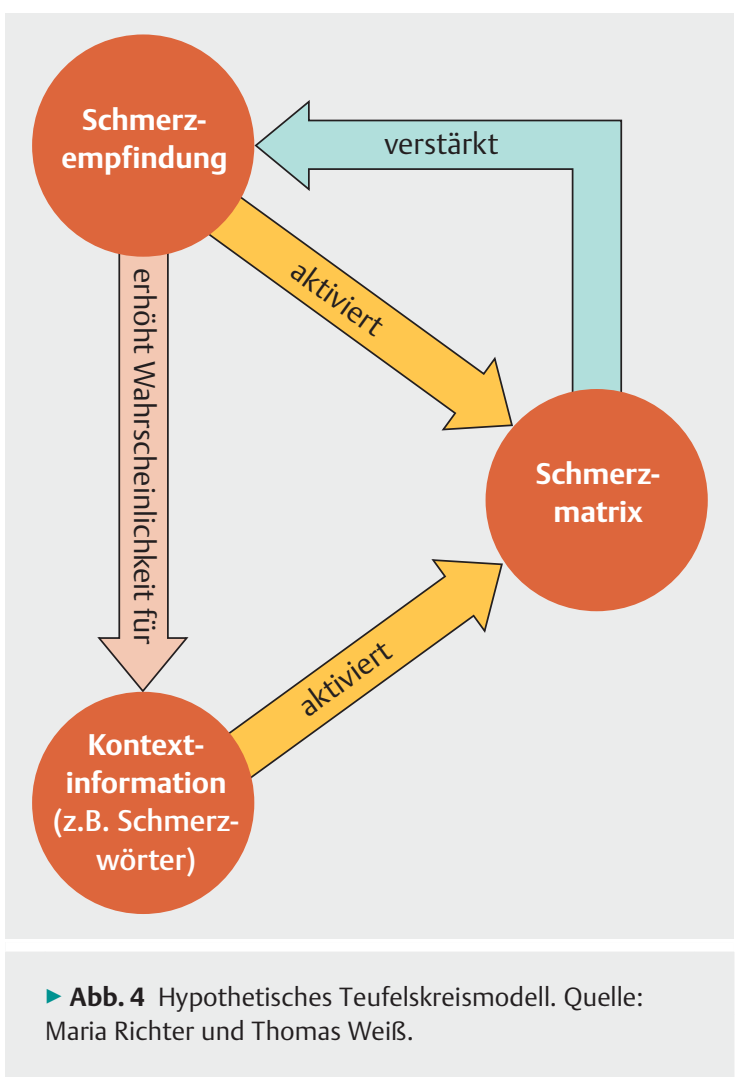

die Assoziation zwischen der aktuellen Lebenssituation und einer ähnlichen Situation, in der bereits Schmerzen erfahren wurden. So verbinden viele der Betroffenen bestimmte Bewegungen mit Schmerzen.

Vulnerabilität In den angeführten kommunikativen Situationen sind Menschen mit chronischem Schmerzgeschehen also vulnerabler für nozizeptive Reize. Außerdem nehmen sie durch die verbale Vorreizung ihren augenblicklichen Schmerz intensiver wahr. Es ist sogar denkbar, dass durch das semantische Priming auch die Schmerzschwelle bei den Betroffenen reduziert wird. Ähnliche Prozesse wurden bereits für Nadeldruckreize bei chronischen Rückenschmerzpatienten beschrieben $[13,14]$.

Abwärtsspirale Wird nun also ein nozizeptiver Reiz durch die Kommunikation von Schmerzwörtern als schmerzhafter empfunden, verstärkt dies wiederum die Aktivierung der Schmerzmatrix, und schmerzassoziierte Kontextfaktoren wie etwa die Schmerzwörter werden in der Folge ebenso stärker verarbeitet: der sich schlieBende Teufelskreis wird so peu à peu zur Abwärtsspirale.

Allerdings ist bislang nicht geklärt, wie stark die Effekte dieser Vorverarbeitung sind. Zudem ist nur in Anfängen bekannt, welche weiteren Einflussfaktoren eine Rolle spielen - etwa der zeitliche Abstand zwischen der Vorreizung und dem Schmerzreiz oder die Intensität der Stimulation [16]. 


\section{Fazit}

Der Einfluss von Sprache auf die Schmerzwahrnehmung ist ein wichtiger Aspekt der Schmerzforschung, der bislang zu wenig Beachtung fand. Wenn man bedenkt, dass sich Patienten im Verlauf von Diagnostik und Therapie in vielfältigen Kommunikationssituationen wiederfinden, sei es während der Schmerzanamnese oder beim Ausfüllen ausführlicher Schmerzfragebögen bei Ärzten oder Physiotherapeuten, so wird schnell die Notwendigkeit weiterer und vertiefender wissenschaftlicher Studien deutlich.

Schmerz-Kommunikation Es bleibt zu diskutieren, welche Art von Schmerz-Kommunikation mit Patienten in welchem Umfang tatsächlich sinnvoll ist und inwieweit das Schmerzgeschehen der Betroffenen dadurch zusätzlich verstärkt wird. Aus klinischer Sicht ist davon auszugehen, dass eine dezidierte Diagnosestellung für die Entwicklung eines optimalen Behandlungsplans und für den Aufbau einer tragfähigen Therapiebeziehung unabdingbar ist. Schließlich sollte sich ein Patient mit seinem Schmerz ernst genommen fühlen und muss hierzu über seine Beschwerden sprechen. Möglicherweise verstärken diese Gespräche aber die Aktivität der Schmerzmatrix und verstärken so die Schmerzen des Patienten. Am Anfang der Behandlung ist diese „Nebenwirkung“ im Vergleich zu den vielfältigen positiven Wirkungen einer ausführlichen Diagnostik und Aufklärung über den Schmerz noch als unproblematisch einzuschätzen. In längeren Behandlungskontexten oder auch im sozialen Umfeld kann eine unverhältnismäßige Schmerzfokussierung in der Kommunikation jedoch durchaus zu einem relevanten Schmerzverstärker werden.

De-Fokussierung Angesichts der Untersuchungsergebnisse erscheint es als sinnvoll und dem Therapieziel dienlich, in längeren Behandlungsverläufen schrittweise auf schmerzbezogene Begriffe zu verzichten und die Aufmerksamkeit auf andere körperliche und emotionale Wahrnehmungsbereiche zu lenken.

So kann etwa zu Beginn einer physiotherapeutischen Therapieeinheit nach dem allgemeinen Wohlbefinden oder zwischenzeitlichen Erfolgserlebnissen anstatt nach dem Schmerzgeschehen gefragt werden. Selbst geringfügige Änderungen im therapeutischen Kommunikationsstil können den Fokus des Patienten deutlich verändern und einer weiteren iatrogenen Chronifizierung vorbeugen. So bietet sich anstatt der obligaten Frage „Wie ist der Schmerz gerade?" eine andere Formulierung an wie z. B. „Wann hat sich ihr Rücken zuletzt gemeldet?“ oder „Wo spüren Sie eine angenehme Veränderung?“.
Hierbei kann es - eine gute therapeutische Beziehung vorausgesetzt - Sinn machen, die Betroffenen im Sinne der Patienten-Edukation über derartige Prozesse aufzuklären, um ihnen so die Chance zu geben, die eigene Kommunikation „schmerz-ärmer“ zu gestalten.

Autorinnen/Autoren

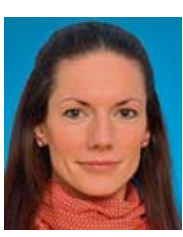

\section{Maria Richter}

promovierte nach ihrem Psychologie-Studium zum Thema „Verarbeitung von schmerzassoziierten Wörtern“ und arbeitet seit 2013 als Psychologische Psychotherapeutin in der Interdisziplinären Tagesklinik für Schmerztherapie am Universitätsklinikum in Jena.

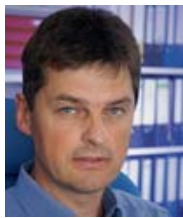

\section{Thomas Weiß}

ist Facharzt für Physiologie (Neurophysiologie) und Professor am Lehrstuhl für klinische Psychologie der Friedrich-Schiller-Universität Jena. Er betreibt intensive Grundlagenforschung zum Thema Schmerz, beispielsweise zur Bedeutung der Sprache für die Schmerzwahrnehmung.

Korrespondenzadresse

Maria Richter
Universitätsklinikum Jena
Klinik für Anästhesiologie und Intensivmedizin
Sektion Schmerztherapie
Am Klinikum 1
07747 Jena
maria.richter@med.uni-jena.de

Korrespondenzadresse

Thomas Weiß

Friedrich-Schiller-Universität Jena

Klinische Psychologie

Am Steiger 3 - Haus 1

07743 Jena

thomas.weiss@uni-jena.de

\section{Literatur}

[1] Apkarian AV, Bushnell MC, Treede R-D et al. Human brain mechanisms of pain perception and regulation in health and disease. Eur J Pain 2005; 9: 463-484. doi:10.1016/j.ejpain. 2004.11.001

[2] Dillmann J, Miltner WHR, Weiss T. The influence of semantic priming on event-related potentials to painful laser-heat stimuli in humans. Neurosci Lett 2000; 284: 53-56. doi:10.1016/S0304-3940(00)00957-5

[3] Eck J, Richter M, Straube T et al. Affective brain regions are activated during the processing of pain-related words in migraine patients. Pain 2011; 125: 1104-1113. doi:10.1016/j. pain.2011.01.026

[4] Hebb DO. The organization of behavior. New York: Wiley, 1949

[5] Iannetti GD, Mouraux A. From the neuromatrix of the pain matrix (and back). Exp Brain Res 2010; 205: 1-12. doi:10.1007/s00221-010-2340-1 
[6] Kenntner-Mabiala R, Pauli P. Affective modulation of brain potentials to painful and nonpainful stimuli. Psychophysiology 2005; 42: 559-567. doi:10.1111/j.1469-8986.2005.00310.x

[7] Kenntner-Mabiala R, Weyers P, Pauli P. Independent effects of emotion and attention on sensory and affective pain perception. Cogn Emot 2007; 21: 1615-1629. doi:10.1080/ 02699930701252249

[8] Lautenbacher S. Commentary to "Do words hurt? Brain activation during the processing of pain words". Pain 2010; 148 : 179. doi:10.1016/j.pain.2009.09.029

[9] Loeser JD, Treede R-D. The Kyoto protocol of IASP Basic Pain Terminology. Pain 2008; 137: 473-477. doi:10.1016/j.pain. 2008.04.025

[10] Miltner WHR, Weiss T. Brain electrical correlates of pain processing. Z Rheumatol 1998; 57 (Suppl 2): 14-18

[11] Peyron R, Laurent B, Garcia-Larrea L. Functional imaging of brain responses to pain. A review and meta-analysis. Neurophysiol Clin - Clin Neurophysiol 2000; 30: 263-288

[12] Pulvermüller F. Active perception: sensorimotor circits as a cortical baiss for language. Nat Rev Neurosci 2010; 11: 351 360. doi: $10.1038 / \mathrm{nrn} 2811$

[13] Puta C, Schulz B, Schoeler S et al. Enhanced sensitivity to punctate painful stimuli in female patients with chronic low back pain. BMC Neurology 2012; 12: 98. doi:10.1186/14712377-12-98

[14] Puta C, Schulz B, Schoeler S et al. Sensory abnormalities for painful and innocuous stimuli at the back and at a site distinct from the region of pain in chronic low back pain patients. PLoS One 2013; 8 (3): e58885. doi:10.1371/journal.pone.0058885

[15] Richter M, Eck J, Straube T et al. Do words hurt? Brain activation during explicit and implicit processing of pain words. Pain 2010; 148: 198-205. doi:10.1016/j.pain.2009.08.009

[16] Richter M, Schroeter C, Puensch T et al. Pain-related and negative semantic priming enhances perceived pain intensity.
Pain Res Manag 2014; 19 (2): 69-74. doi:10.1155/2014/ 425321

[17] Ritter A, Franz M, Puta C et al. Enhanced brain responses to pain-related words in chronic back pain patients and their modulation by current pain. Healthcare (Basel) 2016; 4 (3): 54. doi:10.3390/healthcare4030054

[18] Roa Romero Y, Straube T, Nitsch A et al. Interaction between stimulus intensity and perceptual load in the attentional control of pain. Pain 2013; 154 (1): 135-140. doi:10.1016/j. pain.2012.10.003

[19] Singer T, Seymour B, O’Doherty J et al. Empathy for pain involves the affective but not sensory components of pain. Science 2004; 303 (5661): 1157-1162. doi:10.1126/science. 1093535

[20] Vogt BA. Pain and emotion interactions in subregions of the cingulate gyrus. Nat Rev Neurosci 2005; 6 (7): 533-544. doi:10.1038/nrn1704

[21] Wager TD, Rilling JK, Smith EE et al. Placebo-induced changes in $\mathrm{FMRI}$ in the anticipation and experience of pain. Science 2004; 303 (5661): 1162-1167. doi:10.1126/science.1093065

[22] Weiss T. Plasticity and cortical reorganization associated with pain. Z Psychol 2016; 224: 71-79. doi:10.1027/2151-2604/ a000241

[23] Weiss T, Miltner WHR, Dillmann J. The influence of semantic priming on event-related potentials to painful laser-heat stimuli in migraine patients. Neurosci Lett 2003; 340 (2): $135-$ 138. doi:10.1016/S0304-3940(03)00103-4

Bibliografie

DOI https://doi.org/10.1055/a-0641-7376

Der Schmerzpatient 2018; 1: 168-175

(c) Georg Thieme Verlag KG Stuttgart · New York

ISSN 2512-6210 